\title{
Microfocal radiographic examination of erosions in the wrist and hand of patients with rheumatoid arthritis
}

\author{
J. C. B UCKL AND-W R I GHT \\ From the Macroradiographic Research Unit, Department of Anatomy, Guy's Hospital Medical School, \\ London Bridge, London SE1 9RT
}

SUMMARY The microfocal $x$-ray unit, a new type of $x$-ray machine characterised by an extremely small $x$-ray source, enables great detail to be recorded in the projection radiographs owing to the high object magnification and resolution obtained with this system. Microfocal radiographic examinations were carried out on the wrists and hands of 26 patients with early or moderately advanced rheumatoid arthritis. The macroradiographs confirmed the presence or absence of erosions in patients with early disease activity who otherwise had no detectable lesions visible in standard radiographs. The site of onset, development, and distribution of erosions are described. Their approximate order of appearance is listed. The first to appear occurred in the medial carpal bones, second and third metacarpophalangeal joints and third and fourth proximal interphalangeal joints. In origin they were subperiosteal either immediately adjacent to the insertion of an interosseous ligament or at the transitional zone of the articular cartilage. Erosions in the subchondral bone appeared subsequently and were either indirect (subperiosteal) or direct (subchondral) in origin. Cyst formation was frequently associated with the enlargement of an erosion which it often replaced.

The radiographic examination of the hands of patients with rhe umatoid arthritis described here has been carried out with an entirely new $x$-ray machine-the microfocal $x$-ray unit. ${ }^{1}$ This $x$-ray unit is characterised by an extremely small $x$-ray source, namely, $6-8 \mu \mathrm{m},{ }^{23}$ providing considerable advantages over conventional clinical $x$-ray units. Firstly, a high magnification, $\times 10$ or more, is obtained by placing the specimen close to the $x$-ray source and the film at some distance away. The magnification is the ratio of source-film to source-object distances. Secondly, high resolution is attained; an object as small as 7-20 $\mu \mathrm{m}$ in diameter can be recorded, depending on the type of $x$-ray film employed. Thirdly, there is minimal penumbral blurring. Fourthly, all planes of the object are in focus; stereopair radiographs provide a 3 -dimensional assessment of the structures when the magnification radiographs are viewed under a stereoscope. Fifthly, the object magnification and resolution are great enough to

Accepted for publication 5 April 1983.

Correspondence to Dr J. C. Buckland-Wright. enable direct measurements to be taken from the magnification radiographs.

The first application of microfocal radiography in the study of arthritis was carried out on experimentally induced inflammatory arthritis in guineapigs. ${ }^{2} 45$ During the time-course study of the arthritis microfocal radiography provided a detailed evaluation of the changes in the joints; features such as erosions and osteoporosis were readily quantified. The success of this study led to an evaluation of the effectiveness of a number of anti-inflammatory and antirheumatic drugs in this guinea-pig model. ${ }^{6}$

The advantages of microfocal radiography relative to conventional $x$-ray methods of assessing disease activity in rheumatoid arthritis are described elsewhere. ${ }^{7}$ It was there stated that the fundamental limitation of clinical radiographs was the poor resolution resulting in bony changes not being detected until well advanced. Consequently alterations in the $x$-ray appearance of lesions were not readily detected within 6 months. The merit of the magnification radiograph is that it confirms the presence or absence of erosions in the wrists and hands of patients who otherwise have no obviously detectable lesions vis- 
ible in the standard radiographs (Fig. 1). As osseous changes can be detected within as short a period as 1 to 3 months, a detailed evaluation of the erosions, their site of origin, and their distribution in the wrist and hand was readily obtained. This paper describes the results of such a study.

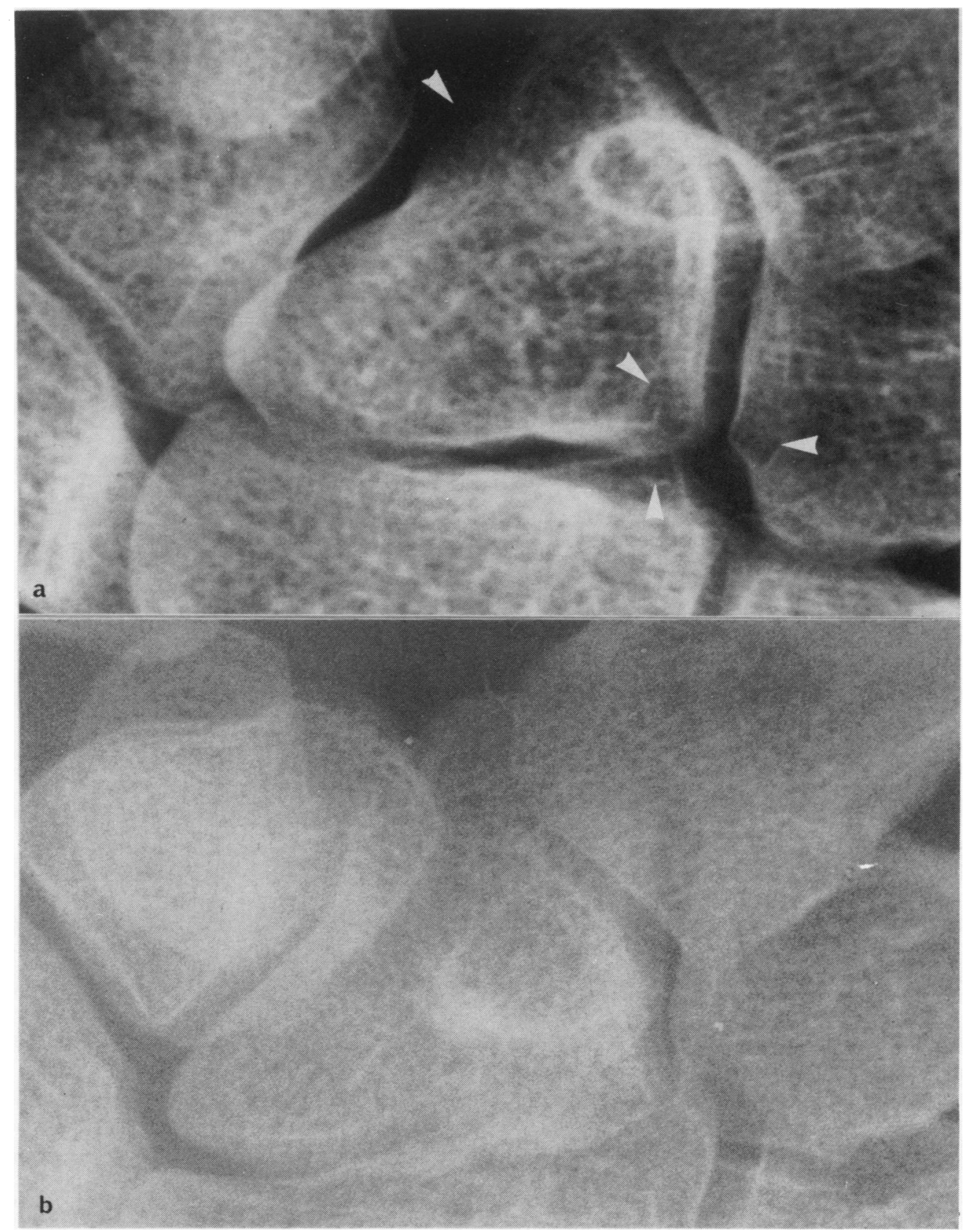

Fig. 1 (a) Part of a magnification radiograph of a patient's wrist with very early rheumatoid arthritis showing the site ofonset of erosions. (b) Radiograph taken with a diagnostic $x$-ray set at the same time and magnified to that of (a). Although the radiographs are not strictly comparable owing to the ulna deviation of the wrist in (b), nevertheless the lesions visible in (a) are not detectable in (b). Primary magnification of $($ a) $\times 5 \cdot 2$, (b) $\times 1$; secondary magnification of $($ b) $\times 5 \cdot 2$. Reproduced $\times 0.9$. 


\section{Patients and methods}

Twenty-six patients with rheumatoid arthritis were divided into 2 groups-those with early and with moderately advanced disease. Patients with early rheumatoid arthritis were referred for microfocal radiographic examination after their first visit to the clinic. They had early clinical evidence of the disease but little or no sign of erosions in the standard radiographs. This group comprised 14 patients, 11 women and 3 men. The mean age at onset of the disease was $41 \cdot 2$ years (range 18-62 years). Twelve patients with moderately advanced rheumatoid arthritis were examined, 5 women and 7 men. The mean age at onset of the disease was 51.7 years (range 32-64 years). Erosions were visible in the clinical radiographs of these patients. Between 2 and 4 sets of radiographs were viewed for each patient taken at either 3 or 6 months apart over a 1- to 2-year period.

\section{MICROFOCAL RADIOGRAPHY}

The microfocal $x$-ray unit, with an $x$-ray source size of $6 \mu \mathrm{m}$ in diameter, was operated at $60 \mathrm{kV}$ and $1 \mathrm{~mA}$. The wrist and hands of the patients were $x$-rayed at $\times$ 5 magnification by placing the part close to the source $30 \mathrm{~cm}$ and the $x$-ray film at some distance away, $1.5 \mathrm{~m}$. With Trimax 16 and 8 screens and XD film (3M, UK, Ltd), the smallest object recorded in the film is of the order of $20 \mu \mathrm{m}$ in diameter, a factor greater than $\times 15$ better than standard radiographs.

The large projection magnification renders it impossible to record the shadow image of the whole hand on a single large $x$-ray plate $(43 \times 35 \mathrm{~cm})$. Therefore separate magnification radiographs were prepared of the wrist joints, the metacarpophalangeal (MCP) joints, and the interphalangeal (IP) joints. The MCP and IP joints of the thumb were omitted, since to have included them would have necessitated either a considerable reduction in the projection magnification, with a resulting loss in radiographic detail, ${ }^{3}$ or the preparation of separate radiographs of just the first MCP and IP joints, with a marked increase in cost.

Stereopair magnification radiographs were prepared at all times. The hand was placed in a stereotaxic unit to keep it still between exposures. The stereotaxic unit and hand were displaced horizontally by $6 \mathrm{~mm}$ between exposures. The stereopair radiographs thus obtained were examined under a largeformat stereoscope for 3-dimensional evaluation of the structural organisation of the bones. During this examination the nature and approximate size of the lesions observed in the wrists and hands of each patient were recorded on data sheets comprising an outline diagram of the bones of the left and right wrists and hands. For each of the different groups of arthritic patients studied a data sheet was compiled summarising the lesion present.

\section{Results}

On the basis of the extent of the lesions recorded in the magnification radiographs of the patients with early rheumatoid arthritis this group was subdivided into those patients with 'very early' and 'early' forms of the disease. The first group comprised 6 women with an average age at onset of the disease of $30 \cdot 2$ years (range 18-52 years) with a mean time interval from the onset of the disease to the first set of radiographs of 9.4 months (range 2 weeks to 2 years). This

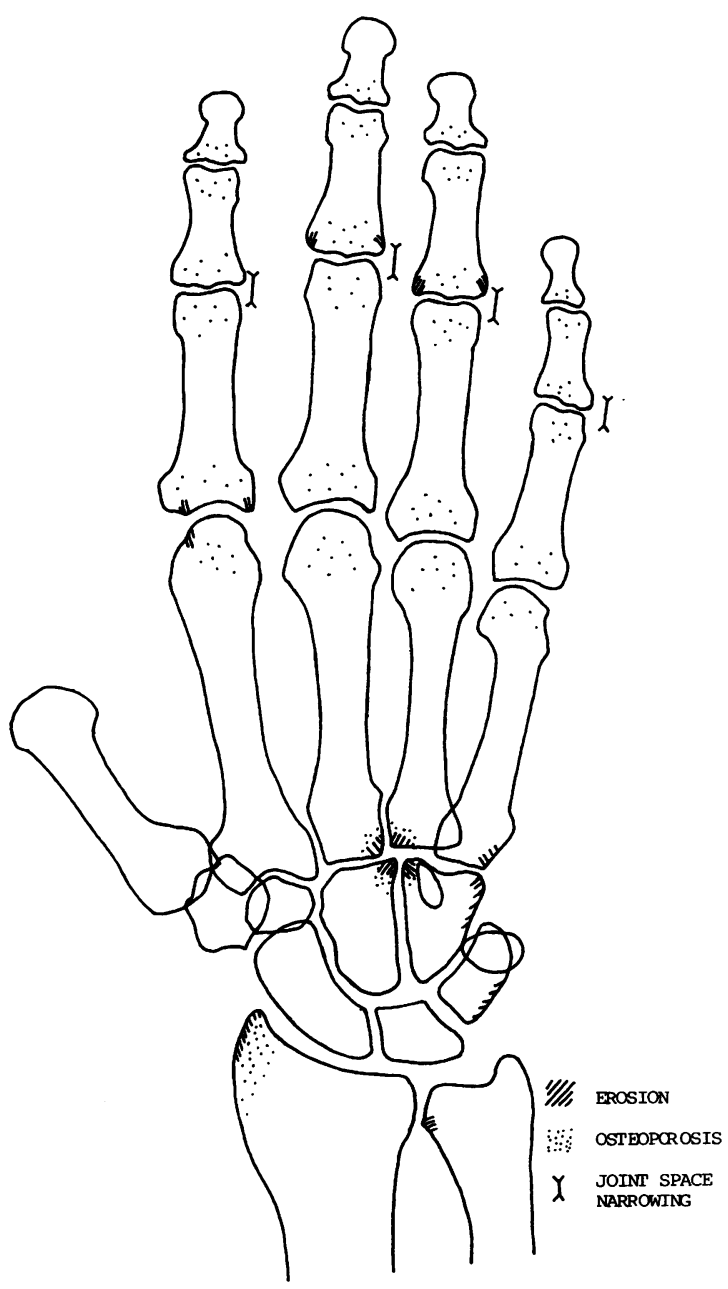

Fig. 2 Diagram of the distribution of lesions in the wrist and hand of patients with 'very early' rheumatoid arthritis. 
group was distinguished by less extensive lesions comprising (1) periarticular subperiosteal erosions, (2) mild juxta-articular osteoporosis, and (3) some loss of joint space. Erosions were detected within 2 months from the onset of the disease. The second group comprised 5 women and 3 men with an average

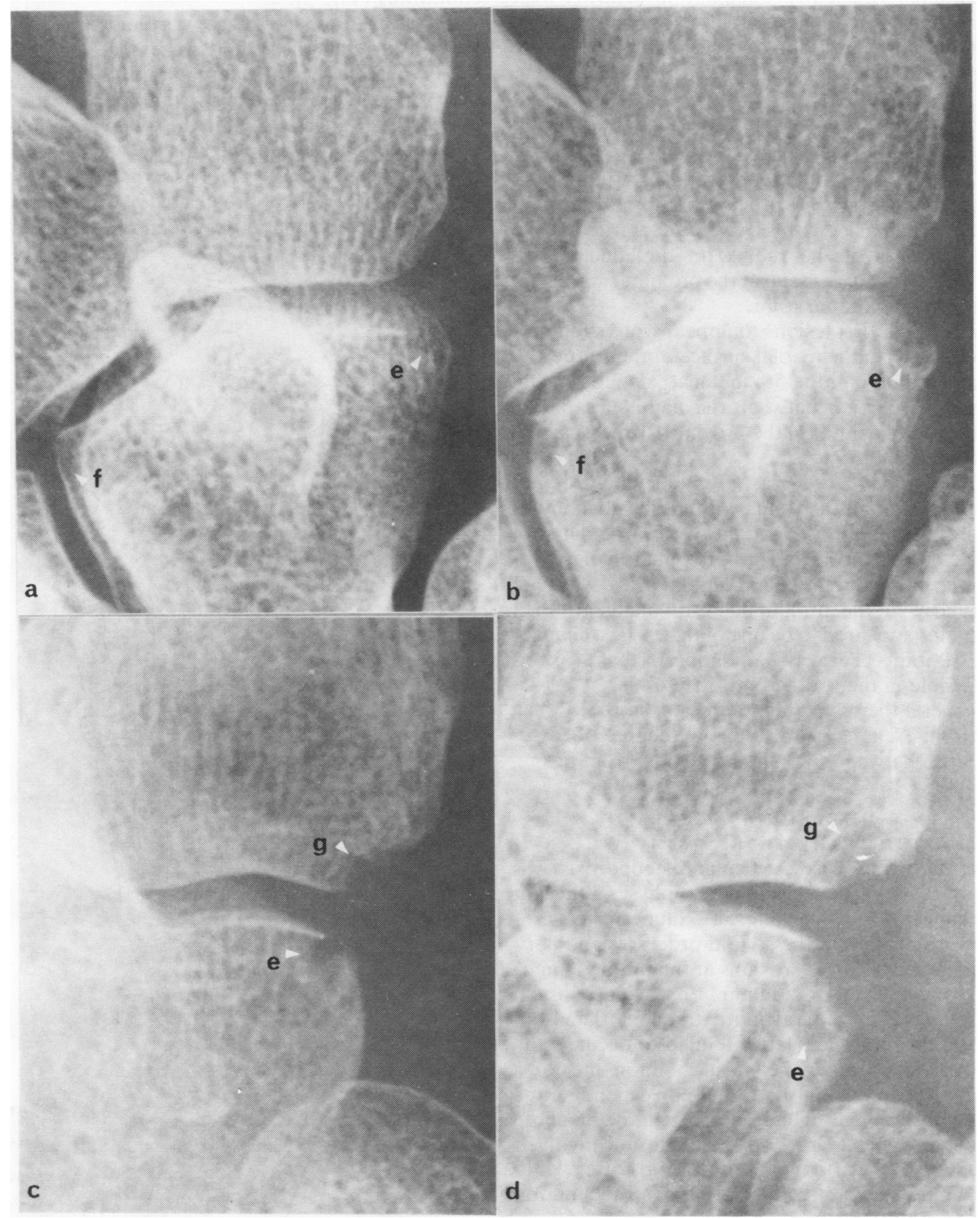

Fig. 3 Part of the magnification radiographs of the wrist of 2 patients showing the onset and development of a periarticular erosion at the disto-medial border of the hamate (e). (a) and (b)are the radiographs of the first patient taken 13 months apart; (c) and (d) those of the second patient taken 12 months apart. Erosion development can be seen at (f) and (g). Primary magnification $\times 5 \cdot 2$, reproduced at $\times 0.9$. 
age at onset of the disease of 49.5 years (range 32 to 62 years) with a mean time interval from the onset of the disease to the first set of radiographs of 16.6 months (range 3 months to 5 years). The lesions in the patients with 'early' rheumatoid arthritis were similar but more advanced affecting many more joints, with the formation of cysts in association with a number of the erosions.

APPEARANCE OF LESIONS IN 'VERY EARLY' RHEUMATOID ARTHRITIS (Fig. 2)

Radius and ulna. Mild juxta-articular osteoporosis was seen in the styloid processes and subchondral bone. Trabecular bone loss was more marked in the styloid process of the radius. In the ulna a subperiosteal erosion was seen at the proximal margin on the inferior radioulnar joint.

Carpus. The first lesions to appear were subperiosteal erosions on the medial surface of the triquetral, hamate, and base of the 5th metacarpal (Fig. 3), and also at the junction between the hamate, capitate, and the proximal ends of the 3rd and 4th metacarpals (MCs) (Fig. 1).

$M C P$ joints. There was a mild juxta-articular osteoporosis in all the joints with early subchondral erosions in the 2 nd MC and early periarticular subperiosteal erosions in the 2 nd proximal phalanx.

IP joints. Slight to mild loss of joint space was seen particularly in the proximal interphalangeal (PIP) joints. This appeared to be one of the earliest changes in the IP joints. Periarticular subperiosteal erosions were visible in the 3rd and 4th PIP joints.

In general there were more erosions in the IP than MCP joints. There was also a good degree of correspondence between the left and right extremities.

APPEARANCE OF LESIONS IN 'EARLY' RHEUMATOID ARTHRITIS (Fig. 4)

Radius and ulna. Juxta-articular osteoporosis was more marked, particularly in the epiphyses, adjacent to the subchondral bone (Fig. 5a). Subperiosteal erosions were present at the styloid processes and periarticular subperiosteal erosions at the proximal margins of the inferior radioulnar joint. Subperiosteal erosions were in general more frequent than subchondral erosions. The latter tended to occur in the ulnar styloid process. The most common site for a subchondral erosion in the radius corresponded to a point opposite the articulation between the scaphoid and lunate bones (Figs. 5, 6). This lesion involved destruction of the articular cartilage and cortex, leading at a later stage to the formation of a subchondral cyst (Fig. 5b).

Carpus. On the medial side of the wrist marked subperiosteal erosions were present in the hands of all the patients, involving the styloid process of the ulna, the medial surface of the triquetral, pisiform, hamate, and the base of the 5th MC. Erosive cysts formed particularly in the last 2 bones at the periarticular margin, with a tendency to extend subchondrally (Fig. 3).

On the lateral side of the wrist subperiosteal erosions occurred in the styloid process of the radius and the lateral surfaces of the scaphoid, trapezium, and base of the 1st MC. No cysts were seen.

Erosions were also seen in the following bones: (1) at the junction between the hamate, capitate, and the base of the 3rd and 4th MCs, where the erosions frequently developed into cysts; (2) at the proximal region of the articulation between the scaphoid and

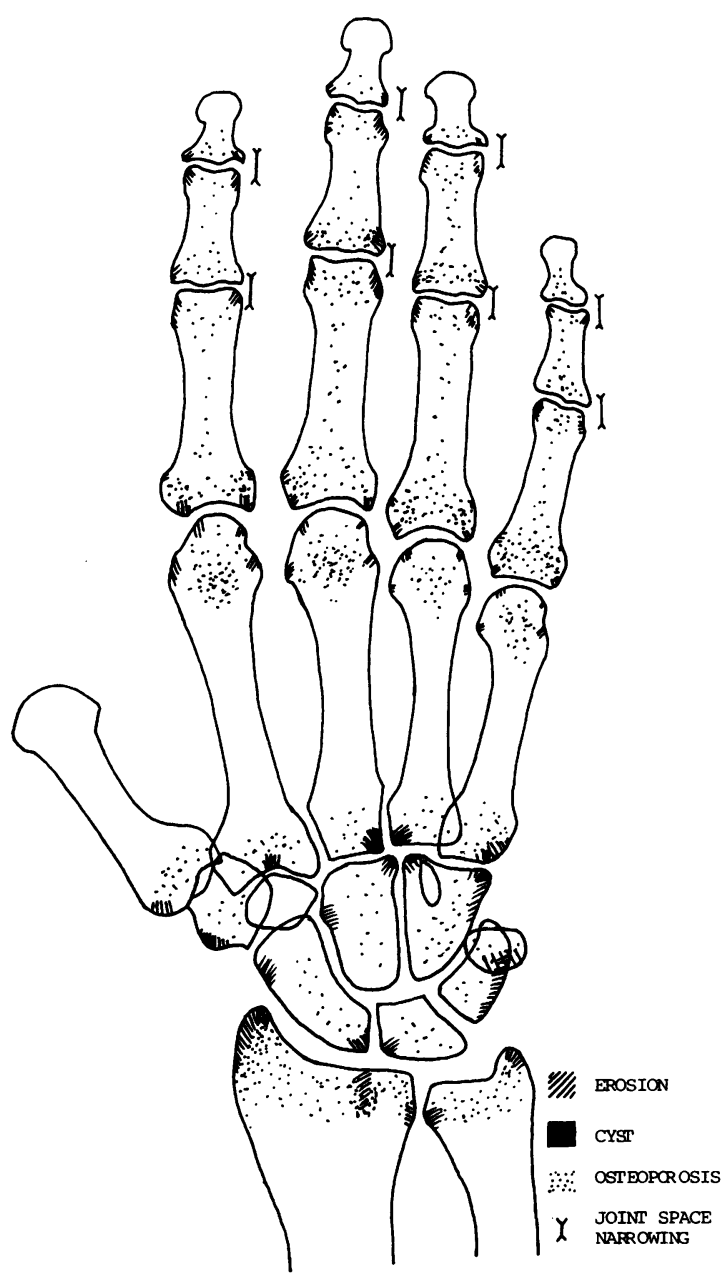

Fig. 4 Diagram of the distribution oflesions in the wrist and hand of patients with 'early' rheumatoid arthritis. 
lunate (Figs. 5, 6); (3) at the distolateral or lateral surface of the capitate close to its articulation with the scaphoid; (4) at the base of the 2nd MC in its mid- articular region. In general osteoporosis was present to some extent in the carpal bones and proximal ends of the MCs.

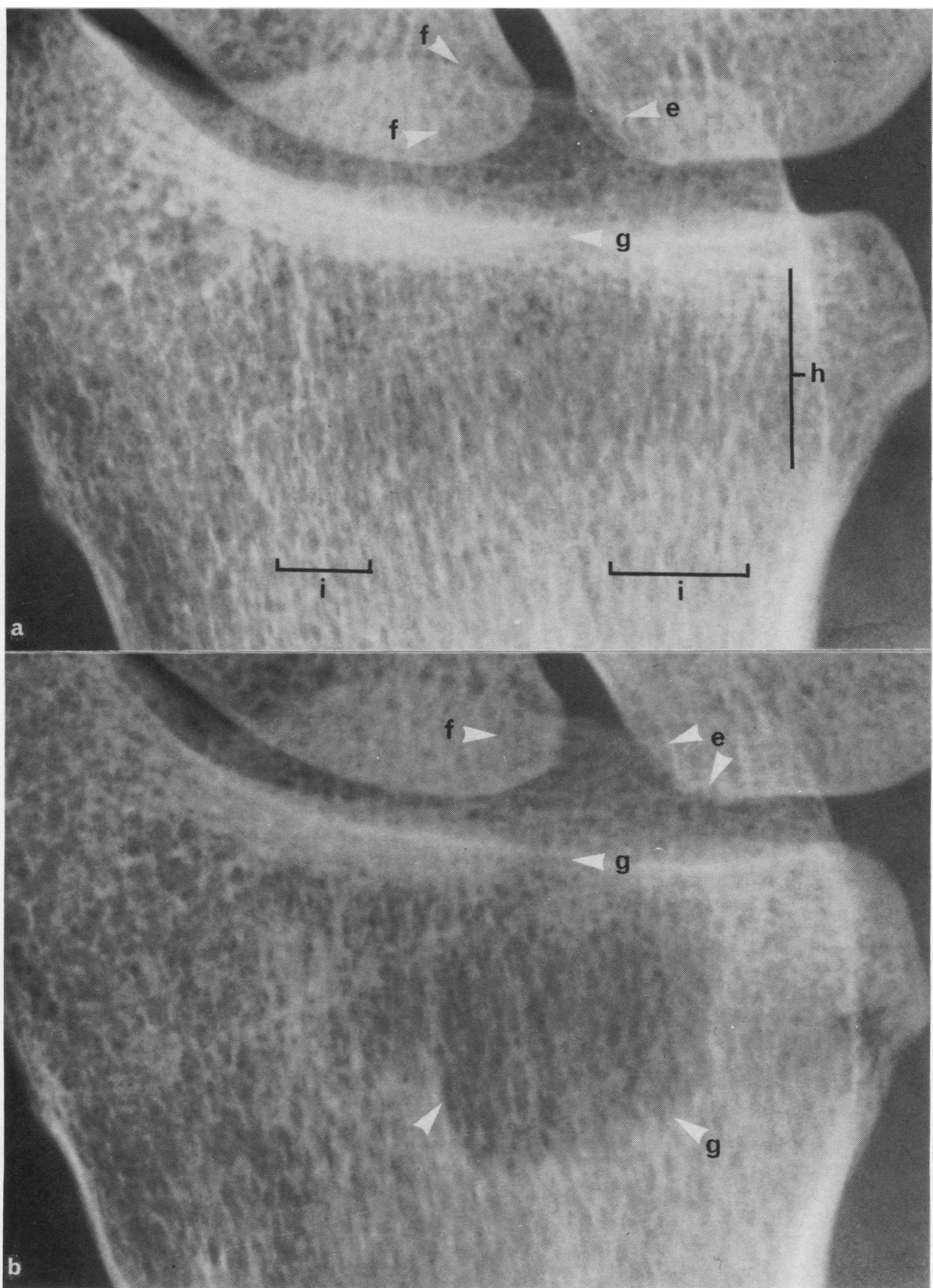

Fig. 5 Part of the magnification radiographs of the wrist of a patient taken 13 months apart showing erosions developing in the lunate (e) and scaphoid $(\mathrm{f})$ and associated subchondral erosion in the radius $(\mathrm{a}, \mathrm{g})$, leading to cyst formation $(\mathrm{b}, \mathrm{g})$; presence of juxta-articular osteoporosis $(\mathrm{a}, \mathrm{h})$ and the retention of force-bearing trabeculae $(\mathrm{a}, \mathrm{i})$. Primary magnification $\times 5 \cdot 2$, reproduced $\times 0 \cdot 8$. 
$M C P$ joints. Juxta-articular osteoporosis was greater than in the wrist. Subperiosteal erosions had developed at the sites of synovial reflection (Fig. 7).
In a few cases small cysts were present. In all of the MCs subchondral erosions had formed, of which a few were quite large.

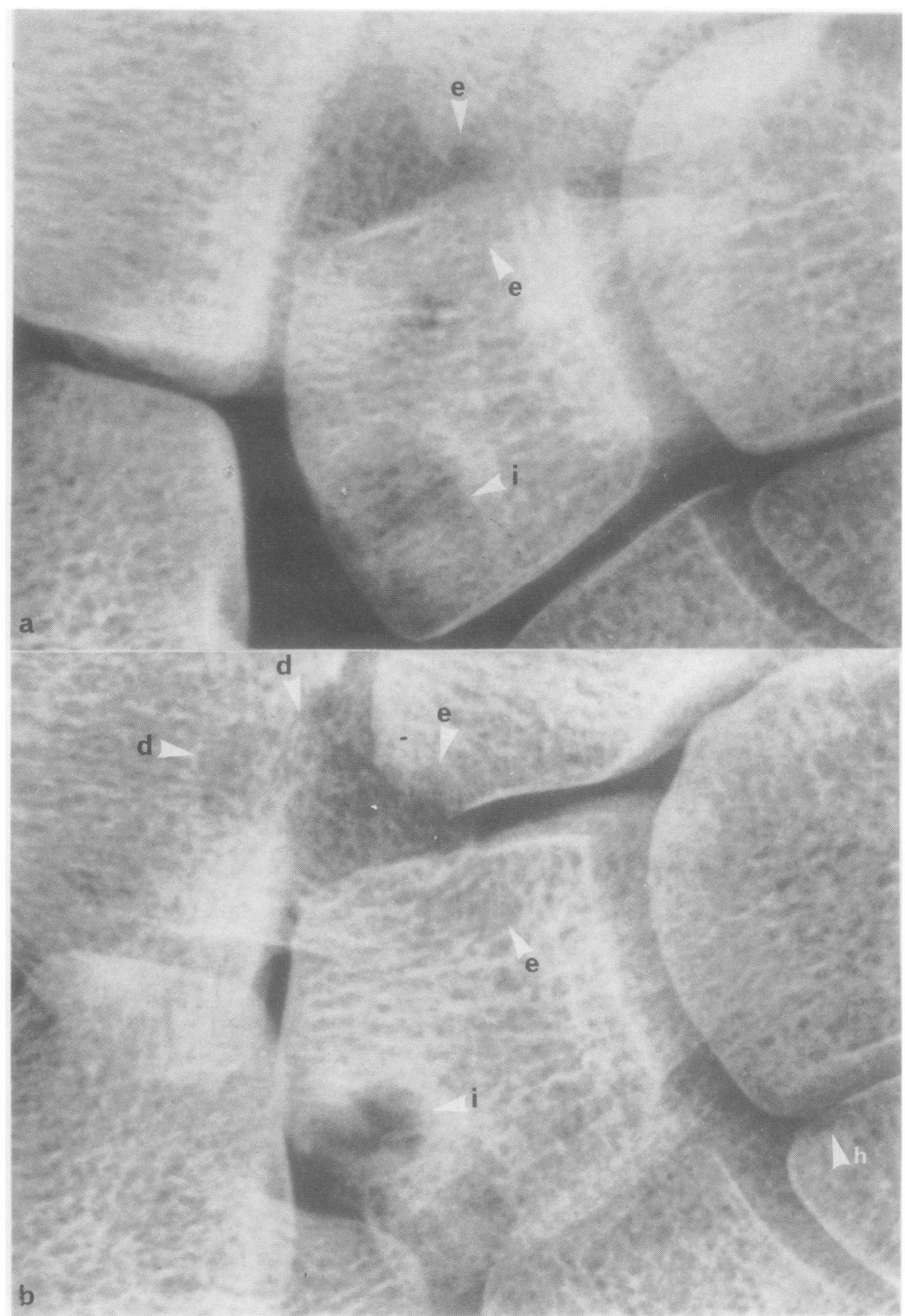

Fig. 6 Part of the magnification radiographs (a) and (b) of the wrist of a patient taken 15 months apart showing the development of (i) an 'indirect' subchondral erosion in the lunate and (d) a 'direct' subchondral erosion in the radius with erosions in the scaphoid and lunate (e). A subchondral erosion is present at the proximal end of the hamate (h). Primary magnification $\times 5 \cdot 2$, reproduced $\times 0 \cdot 8$. 
$I P$ joints. The changes at these joints were occasionally associated with those of a degenerative joint disease. Joint space narrowing was seen at all the joints as well as juxta-articular osteoporosis. Subperiosteal erosions were seen at nearly all the joints at the periarticular margin and synovial reflection. In
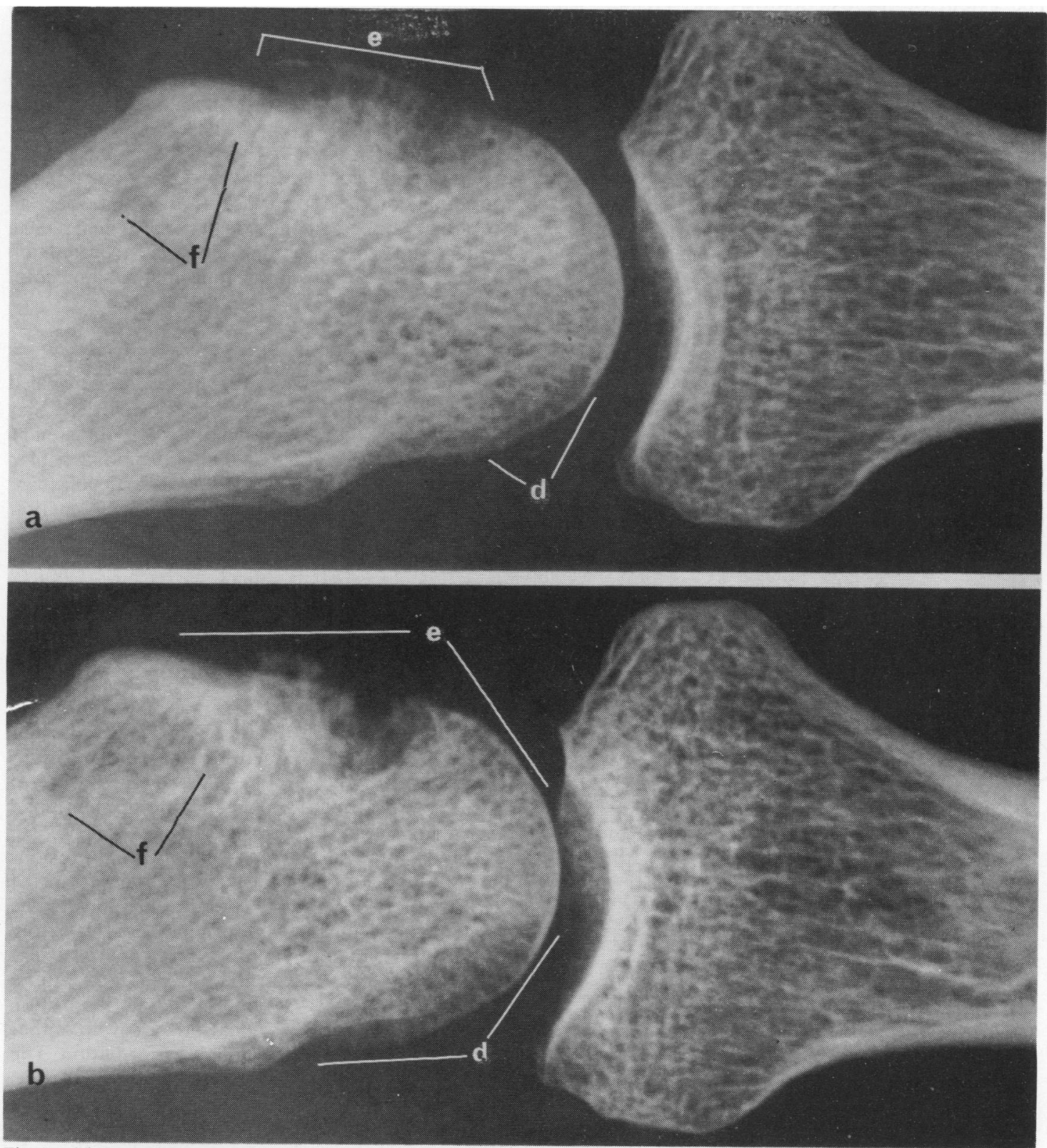

Fig. 7 (a) Part of a magnification radiograph of the MCP joints of a patient. The 2 nd MCP joint shows small periarticular erosions at (d) on the me dial side and a large erosion with early cyst formation at (e). A subperiosteal erosion proximal to the attachment of the collateral ligament is visible at (f). (b) Part of a magnification radiograph showing the same joint taken 3 months later. The erosions and cyst have enlarged. There is an increase in the juxta-articular osteoporosis. Primary magnification $\times 4 \cdot 7$, reproduced at the same magnification. 
general the extent of the erosions was greater than those seen in the MCPs.

\section{APPEARANCE OF LESIONS IN MODERATELY}

ADVANCED RHEUMATOID ARTHRITIS (Fig. 8)

The extent of the osteoporosis was greater, with marked loss of trabeculae in the epiphyses. However, within this same region the large force-bearing trabeculae appeared to be retained (Fig. 5). The major difference in the bony lesions between this group and those with less severe arthritis was the greater extent of destruction of the subchondral bone on the former. In many instances erosions had developed into cysts (Fig. 7).

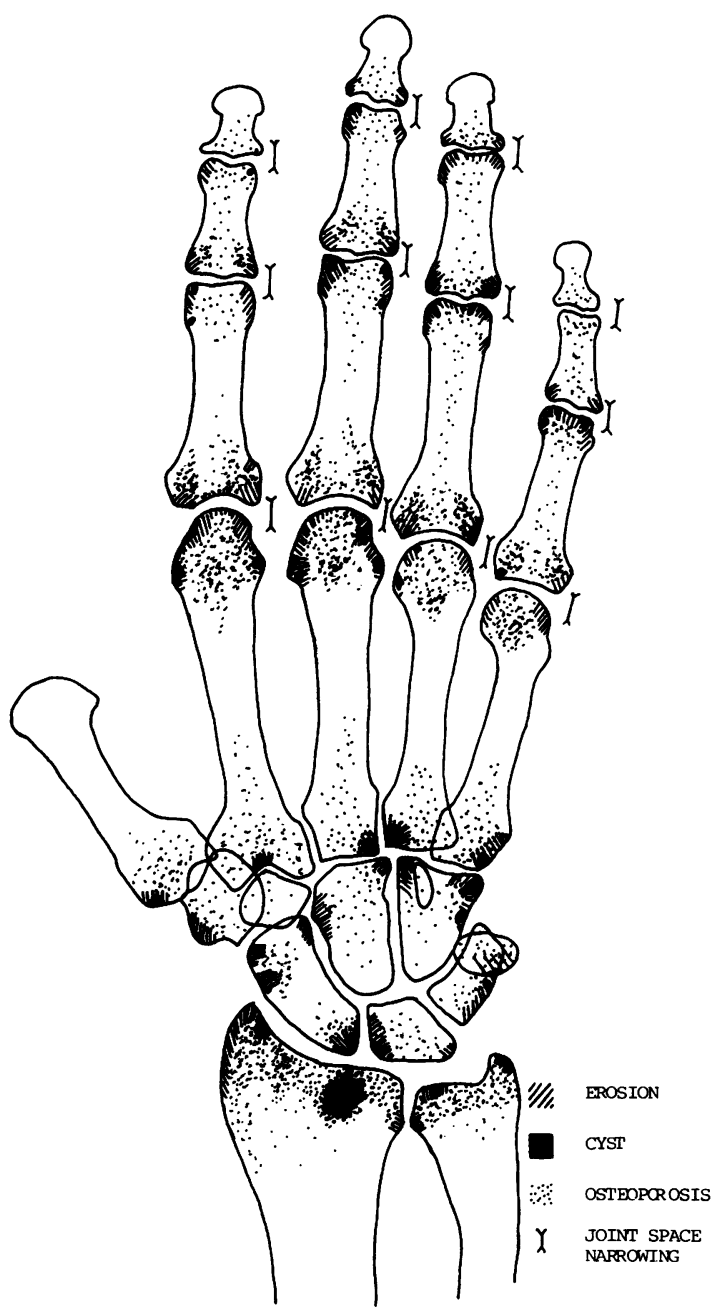

Fig. 8 Diagram of the distribution oflesions in the wrist and hand of patients with moderately advanced rheumatoid arthritis.
Wrists. Juxta-articular osteoporosis in the epiphyses of the radius and ulna was generally marked. In particular the removal of the trabeculae in the epiphyseal region so as to produce the effect of a zone of osteoporosis relative to the denser trabeculation in the metaphyses was noteworthy (Fig. 5a). In the radius subchondral bone was lost from the region of the styloid process and at the point opposite the articulation between the scaphoid and lunate leading to the disruption of the cortical plate and the formation of a cyst (Fig. 5).

$M C P$ and IP joints. Apart from the increase in the extent of the lesions a trend was observed within the formation of some of the erosions. In a joint where an erosion developed on one side of the proximal bone a similar and corresponding erosion occurred on the same side in the distal bone of the same joint (Fig. 9).

\section{EROSION AND CYST FORMATION}

Examination of the magnification radiographs revealed that the first erosions were subperiosteal and that the site of origin corresponded directly to the area of reflection of the synovium on to the nonarticular surface of the epiphysis and at the periarticular margin, i.e., the transitional fibrocartilaginous junction between the articular cartilage and the periosteum.

The erosions appeared as localised areas of radiolucency due to the reduction in thickness and/or removal of trabeculae (Figs. 3, 7). The overlying cortical bone was thin. In the early stages the site of entry of the pannus could occasionally be seen as a small perforation in the cortex, $0 \cdot 1-0 \cdot 2 \mathrm{~mm}$ diameter. As the erosion progressed it increased in size, and the subperiosteal cortex and trabeculae were further destroyed with a consequent disruption of the configuration of the bone (Figs. 3, 7). The majority of erosive cysts formed as a result of the continued destruction of the subperiosteal bone (Fig. 7).

Further confirmation that subperiosteal erosions were the first lesions to occur was observed in the MCP joints. Here, the subchondral erosions at the distal ends of the MCs were originally derived from the distal migration of pannus from the periarticular subperiosteal erosions at the medial and lateral epicondylar surfaces, to the subchondral position (Fig. 7). Subchondral erosions and cysts were either indirect or direct in their manner of formation. The first to appear were indirect as described above, where the subperiosteal pannus migrated to a subchondral region (Fig. 6). The direct type occurred later as a result of the pannus invading the subchondral bone after the destruction of the articular cartilage (Figs 5,6). One of the earliest sites of formation of the direct type of subchondral erosion was in the midarticular region of the radius opposite 


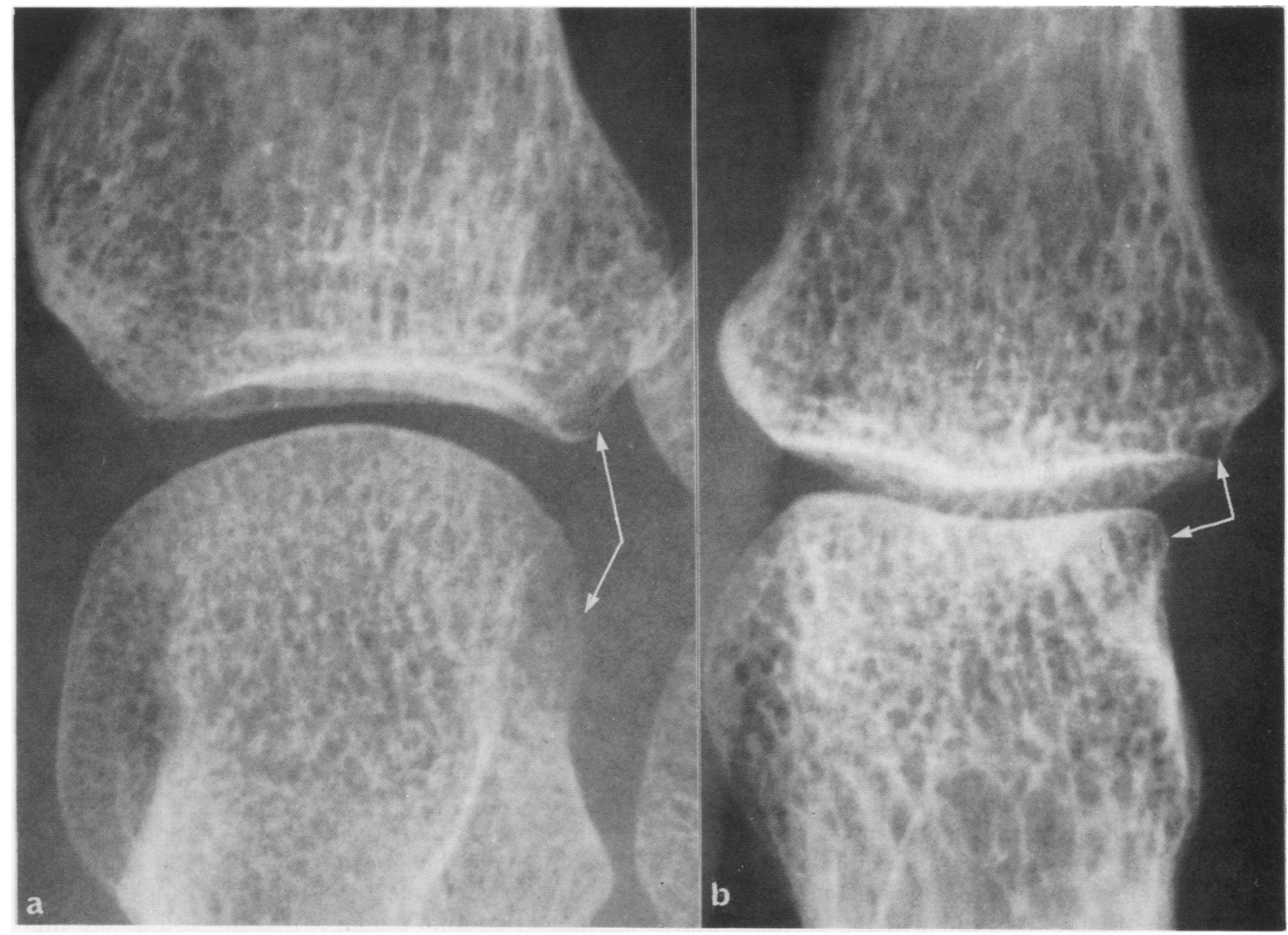

Fig. 9 Part of the magnification radiographs showing (a) the 4th MCP and (b) 4th PIP joints with intra-articular correspondence of erosions on the medial side of the joints.

the articulation between the scaphoid and lunate (Figs. 5, 6).

\section{Discussion}

Microfocal projection radiographic examination of the wrists and hands of patients with rheumatoid arthritis has led to the identification of the sites of onset and progression of erosions. The first to appear were subperiosteal, corresponding directly to the area of reflection of the synovium on to the bone, the majority occurring adjacent to the attachment of the interosseous ligaments (Fig. 10). Of these the first were on the medial side of the wrist (hand held in anatomical position) adjacent to the points of attachment of the medial collateral and interosseous ligaments at the ulna styloid process, triquetral,

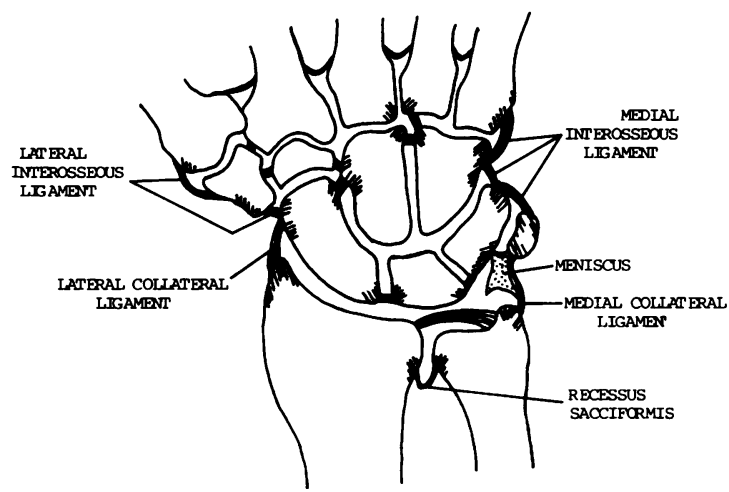

Fig. 10 Diagram of the arrangement of the interosseous ligaments in the wrist and their association with the site of onset of erosions adjacent to their attachment. The hatched areas indicate the site of erosions. 
hamate, and base of the 5th MC. Similarly, on the lateral side of the wrist erosions were observed next to the attachment of the lateral collateral and interosseous ligaments at the styloid process of the radius, the scaphoid, trapezium, and base of the 1st MC. Erosions occurred close to the attachment of the interosseous ligament between the lunate and scaphoid, on the lateral surface of the capitate adjacent to the ligaments extending from the capitate to the trapezoid and scaphoid. The erosions at the junction between the hamate, capitate, and the base of the 3rd and 4th MCs were associated with the hamate-capitate and hamate-base of 4th MC interosseous ligaments and the palmar ligaments attached to the 4 bones. In the MCP and IP joints subperiosteal erosions appeared at the synovial reflection adjacent to the points of attachment of the collateral ligaments and at the periarticular margins (Figs. 7, 9).

Subperiosteal erosions not directly associated with ligamentous attachments were seen at the periarticular margins of the MCP and IP joints and at the articular margin of the inferior radioulnar joint corresponding to the subsynovial region of the joint's bursa (recessus sacciformis).

Two types of subchondral erosion, 'indirect' and 'direct,' were identified. The first to form were the 'indirect,' in which a periarticular subperiosteal erosion extended to a subchondral region (Figs. 6, 7). The 'direct' subchondral erosions occurred later as a result of the disruption of the articular cartilage and the invasion of the pannus into the subchondral bone. One of the earliest to appear in the wrist was at the midarticular region of the radius opposite the scapho-lunate articulation (Figs. 5, 6). It is possible that a concentration of pannus in this region, associated with the erosions in these 2 bones, may have accelerated the destruction of the articular cartilage and subchondral bone of the radius, ultimately leading to the formation of a cyst (Fig. 5). The extent of the cyst within the epiphysis was limited to some degree by the retention of the load-bearing trabeculae on either side of the cyst. These trabeculae are associated with the transmission of force from the scaphoid and lunate respectively (Fig. 5).

On the basis of the present results Table 1 lists the approximate order of appearance of erosions in the wrists and hands of patients with early rheumatoid arthritis. As nearly all clinical radiographs are taken of both hands in ulnar deviation, the earliest erosions occurring on the medial side of the wrist are obscured by the superimposition of adjacent bones (Fig. 1). It is recommended that if early erosions are to be detected in clinical radiographs the hands should be radiographed without an ulnar deviation.

The distribution of erosions in the MCP and PIP joints of patients with early rheumatoid disease confirms previous observations ${ }^{89}$ of an inverse relationship between the damage at the PIP and MCP joints. This arrangement was not maintained in patients with more advanced disease, where, contrary to the observations of Brook and Corbett, ${ }^{10}$ erosions tended to be more frequent in the IP rather than MCP joints.

The close association between erosions and the attachment of the interosseous ligaments tends to support the role of mechnical factors ${ }^{811}$ in determining the site of joint damage. It is suggested here that with the increase in intra-articular pressure associated with the disease the ligament during joint movement assists in compressing the inflamed synovium against the subsynovial bone, leading to the formation of an erosion. Further, raised intraarticular pressure would account for the migration of

Table 1 Approximate order of the site ofonset of erosions seen in the magnification radiographs of patients with 'very early' and 'early' rheumatoid arthritis

\begin{tabular}{lll}
\hline Group & Wrist & Hand \\
\hline Very early RA & Medial side hamate and base of 5th MC \\
& Junction between hamate, capitate, base 3rd and 4th MCs \\
Medial side triquetral & 2nd and 3rd MCPs & 3rd and 4th PIPs \\
& Styloid side head of ulna (inf. radioulnar joint) & Remaining MCP \\
Early RA & Styloid process of ulna & and IP joints \\
& Medial surface of radius (inf. radioulnar joint) & \\
& Scapho-lunate articular surfaces & \\
& Lateral surface of scaphoid & \\
& Lateral surface of base 1st MC & \\
& Lateral surface of trapezium \\
& Subchondrace of capitate & \\
\hline
\end{tabular}


pannus from a subperiosteal position to the subchondral region, as seen in the formation of indirect subchondral erosions (Figs. 6,7).

A principal factor which may determine the onset of erosions adjacent to the attachment of ligaments and at the periarticular margins is that both these sites are characterised by a subsynovial rich plexus of blood vessels or mesentery. ${ }^{12}$ The vessels in the mesentery are connected to the rich plexus of veins in the synovium and hence to the biochemical activity of this tissue. Humoral degradative factors may well be transported via the venous plexus to the subsynovial mesentery at the attachment of the ligaments and at the periarticular margins. Support for the vascular connection between the synovium and the subsynovial mesentery at the attachment of the collateral ligaments was seen in the MCP and IP joints in the intra-articular correspondence of erosions on the same side of the joint between proximal and distal articular elements (Fig. 9). This phenomenon would also appear to apply to other joints such as in the wrist, between the hamate and base of 5th MC (Fig. 3 ). The subsynovial mesentery is also connected to the large venous sinusoids in the epiphyse ${ }^{12}$ and may well account for the localised subchondral osteoporosis seen in the radius (Fig. 5) and ulna as well as that in the juxta-articular regions of the MCP and IP joints.

The consistency in the site of onset of erosions in relation to joint anatomy strongly suggests that their origin would be similar for all other synovial joints. Thus it would be possible to predict the likely sites of onset of erosions in the different joints of the body. Further, as erosions tend to occur initially in the metatarsophalangeal joints, ${ }^{10}$ it may be possible to predict that erosions are present in these joints when lesions are recorded in the wrist and hand of a patient. These suggestions can be confirmed by the microfocal radiographic examination of the various joints in rheumatoid patients.

I thank Dr R. Grahame, Professor G. Panayi, and Dr T. Gibson for their co-operation in providing the patients for $x$-ray examination, Professor V. Wright and $\mathrm{Dr} \mathrm{H}$. Bird for kindly reading the MS, and Mr K. Fitzpatrick for preparing the photographs.

This work is being generously supported by the Arthritis and Rheumatism Council.

\section{References}

1 Ely R V, ed. Microfocal radiography. London, New York: Academic Press, 1980.

2 Buckland-Wright J C. Qualitative and quantitative assessment of tissue organization in normal and diseased organs. In: Ely R V, ed. Microfocal radiography. Academic Press, London, New York: 1980: 147-95.

3 Buckland-Wright J C. (1983) Microfocal projection radiography, a new diagnostic and investigative technique. $\mathrm{Br} J$ Radiol (submitted for publication).

4 Buckland-Wright J C, Cashin C H, Doherty N S, Jeffries B L. Quantitative assessment of experimentally induced arthritis in guinea-pigs using microfocal radiography. In: Willoughby D A, Giroud J P, eds. Inflammation: mechanisms and treatment. Lancaster: MTP Press, 1980: 429-38.

5 Buckland-Wright J C. Microfocal radiography in the quantitative assessment of experimentally induced inflammatory arthritis in guinea-pigs. $J$ Pathol 1981; 135: 127-45.

6 Cashin C H, Doherty N S, Jeffries B L, Buckland-Wright J C. An investigation into the effect of anti-inflammatory and antirheumatic drugs in cell mediated immune arthritis in guinea-pigs by microfocal radiography. BrJ Exp Pathol 1980; 61: 296-302.

7 Buckland-Wright J C. $X$-ray assessment of activity in rheumatoid disease. BrJ Rheumatol 1983; 22: 3-10.

8 Martel W, Hayes J T, Duff I F. The pattern of bone erosion in the hand and wrist in rheumatoid arthritis. Radiology 1965; 84: 204-14.

9 Mattingly P C, Matheson J A, Dickson R A. The distribution of radiological joint damage in the rheumatoid hand. Rheumatol Rehabil 1979; 18: 142-7.

10 Brook A, Corbett M. Radiographic changes in early rheumatoid disease. Ann Rheum Dis 1977; 36: 71-3.

11 Glick E N. Influence of mechanical factors on the rheumatoid wrist. Proc $R$ Soc Med 1966; 59: 555-8.

12 Brookes M. The blood supply of bone. London: Butterworth, 1971. 